\title{
Digitalization of management: collective behavior modeling on the base of cellular emotional automate
}

\author{
Vladimir Meshkov ${ }^{1}$, Natalia Kochkovaya ${ }^{1, *}$, Irina Usova $^{1}$, and Liudmila Stolyar ${ }^{1}$ \\ ${ }^{1}$ Institute of Technologies (branch) of DSTU in Volgodonsk, 347386, Mira avenue, 16, Volgodonsk, Rostov \\ region, Russia
}

\begin{abstract}
The paper considers the author's approach to digitalization of management of the emotional state of collectives of individuals. Collective models are built on the basis of the «cellular emotional automate» proposed by the authors. The possibility of using the standard rules for cellular automate in modeling the emotional behavior of collectives is shown. The proposed approach can be attributed to artificial intelligence technologies.
\end{abstract}

\section{Introduction}

One of the main tasks of introducing artificial intelligence technologies into modern life is the task of digitalizing the management of both artificial and natural systems. So, today there are many attempts to use a wide variety of natural computing models in the digitalization of management not only for technical systems, but also for human collectives.

The classic sections of natural computing at the moment are: genetic algorithms, cellular automata, artificial neural networks [1-7].

In this work, we will focus on models based on cellular automate. Cellular automates are a discrete system that changes its state step by step using transition rules. We will consider the possibility of constructing «emotional» № cellular automate as models of individuals and models of collectives.

Let's consider the models of collective behavior from the point of view of natural computing.

What is a collective? How is it organized? On what principles can a team be organized? This is either a hierarchy of random interactions, or object modeling, attribute models, etc. It is these issues that we will consider further, taking into account the main paradigm of this article.

By a collective we mean a certain organized set of cellular automate.

It is also worth noting that by management we mean the process of purposeful impact on something or someone. In this case, the impact is directed at the system, which is a collective of individuals (or, more formally, automata).

The choice of a structural model of team management (from hierarchy to anarchy).

There are not so many organizational models of management: it is, first of all, a hierarchical system, centralized, decentralized (described in the work of V.I. Varshavsky and D.A. Pospelov

\footnotetext{
* Corresponding author: kochkovaya@mail.ru
} 
«The orchestra plays without a conductor»), network (in the political aspect - network power, and in the economic [7] - the organization of network marketing, in the computer version online social networks and multi-agent or viral technologies [4]).

Thus, the purpose of control in one case is this or that state of the system. The very required (desired) state is determined, on the one hand, by the considered (or visible) parameters of the system, and on the other hand, the values and vector of these parameters are determined by many factors of higher abstraction (ideology, politics, etc.).

In another case, the purpose of control may consist in the formation of the required parameters of the environment, and such that indirectly ensure the creation and functioning of the «necessary» systems. Thus, it is necessary to form a pair «environment - system». The system is considered as an automaton, and the external environment - as a set of influences (input signals) on a given automaton.

\section{Materials and methods}

In work [7-15], among others, the personal tasks of management are considered (meeting the interests of the head and / or the administrative apparatus).

We will consider just such problems. Then, as the goal of management, we will choose a change or reduction to the desired level of the collective's emotional state.

Then, as an evaluative control function, one can use, for example, the vector of values of the emotional states of individuals (emotional cellular automata [15]), and for the collective as a whole, a generalized vector (some additive function with weight coefficients of the influence of individual automata). The evaluation function can also be formulated as a decision rule for a set of cellular automaty [5].

Management can be built iteratively or continuously. You can form an emotional field in which the goal is achievable. Thus, the vector and the values of the parameters of this vector are formed, which characterize the required state of the system (that is, the goal of control).

At a higher abstract level (the level of the meta system), it is necessary to look at two structural extremes: a hierarchical model, that is, a strict structure, where there is a clear subordination and hierarchy with transcendence in relation, etc., and a random (stochastic) model with the possibility of a superstructure.

In hierarchical systems, the management goal is traditionally understood as the solution of two problems:

- keeping the system in its current state (stabilization task)

- transfer of the system to the required state.

Another structural extreme is a random (stochastic) model with the ability to build on or adjust the interactions of quite autonomous and independent systems, but the behavior of which is imposed by some external environment [9]. Then we can speak about the formation of this external environment as influencing the behavior of a set of automata. In this case, the totality of sets of heterogeneous systems create structural chaos. Consequently, in this case, the purpose of management is to form an appropriate external environment, for example, information multivariate multidimensional virtual reality. Note that modern complex systems, as a rule, are not hierarchical, but structurally random.

\section{Results}

In [15], the concept of «emotional automate» is introduced, which is understood as a discrete automaton, the states of which can be certain emotions.

For example, Timothy Leary [16] has such a concept as imprints, which allow one to represent a certain formalized sequence of influences, maybe it can even be called an algorithm 
of influences that, as a result, generate certain emotions. Then we could consider just imprints as understood by $\mathrm{T}$. Leary as input influences on our emotional automaton and corresponding reactions. Based on this approach, it can be extended to artificial systems as well although in the mentioned work it is more about social systems and about man.

The book [16] provides a classification of emotions in their moderate and extreme manifestations, which can be taken as a basis for constructing emotional automata.

In [15], two approaches to the formation of an emotional automaton are considered.

On the one hand, emotion can be viewed as a kind of output signal, i.e. the input signal and the previous state generate some emotion. For example, the emotion of fear can be triggered by some input signal defining some threshold state close to the destruction of an automaton (object or subject). In such a machine, emotions are converted, i.e. the flow of emotions from one to another. For example, if «Suffering» is a state (emotion), then «loss of inner strength» is an input signal of the automaton, and «feeling of loneliness and self-pity» is an output signal.

In the second approach, it is the internal state of the automaton that is the emotion. Then we must say that emotionality is some global or important state of the system, a total indicator. Moreover, not only for a person, but also for the system as such. And the internal state of the system (emotion) can be changed either by a certain set of input signals, or by applying a certain rule.

When applying the second approach, taking into account a set of rules, we obtain an elementary cellular automaton [1-4].

An example of a state table for such an emotional automaton is given below in the text. This table should be seen as descriptions of the emotional automate and not as a rigorous psychological study or a guide to direct practical action or practical use in psychological practice.

Table 1. Table of states of an emotional automate

\begin{tabular}{|c|c|c|}
\hline State (emotion) & Input signal & $\begin{array}{c}\text { Output signal } \\
\end{array}$ \\
\hline Interest & Motivation to learn & $\begin{array}{l}\text { Attention and interest in the object of } \\
\text { interest }\end{array}$ \\
\hline Joy & Action related to self-confidence & $\begin{array}{l}\text { Realization and / or growth of self- } \\
\text { importance }\end{array}$ \\
\hline Surprise & Unexpected & $\begin{array}{l}\text { Releasing previous emotions and } \\
\text { focusing on the object of surprise }\end{array}$ \\
\hline Suffering & $\begin{array}{l}\text { Standing "loss of inner strength", } \\
\text { pain, fear, horror }\end{array}$ & Feeling of loneliness and self-pity \\
\hline Anger & Reaction to negative event & $\begin{array}{l}\text { Mobilization of energy, feeling of } \\
\text { strength, courage and self-confidence }\end{array}$ \\
\hline Disgust & $\begin{array}{l}\text { An object or phenomenon with } \\
\text { physical }\end{array}$ or $\begin{array}{l}\text { psychological } \\
\text { deterioration, defects in aesthetic } \\
\begin{array}{l}\text { qualities, distortion of } \\
\text { principles }\end{array}\end{array}$ & Desire to get rid of the object \\
\hline Contempt & Meeting a dangerous opponent & Feeling superior \\
\hline Fear & Real or fictitious threat, bad feeling & Lack of confidence \\
\hline Guilt & $\begin{array}{l}\text { Violations of a moral, ethical or } \\
\text { religious nature in situations where a } \\
\text { person feels personally responsible } \\
\text { for the events that occur }\end{array}$ & Feelings of guilt \\
\hline
\end{tabular}

In the future on the basis of the above table, it is necessary to build a transition table for some real or virtual subject. However, the solution to this problem is associated with the study and construction of an emotional portrait of the relevant subject (for example, "athlete" as a concept, a character in a computer game, etc.). This work requires a fairly significant 
investment of intellectual resources and, as a result, will be considered by the authors in another work. Let us emphasize that the construction of a detailed emotional automaton is always multidimensional [6].

Note that an emotional person is able to listen to his own feelings, control emotional impulses and make decisions. All this can be viewed as a switch of an "emotional" automate (or a group of automata).

Then, let an elementary cellular automaton be an emotional automaton [15] of a binary type, that is, there are two stable states, for example, «rest» and «aggression». Then, to control the emotional mood of a certain society, the individuals of which are modeled by such an automaton, it is enough to choose the appropriate rule to achieve the set control goal. The goal may be the desire to reduce social tension.

Consider a number of rules of behavior of cellular automata (according to the classification of Wolfram Stephen Wolfram, Stephen [17]).

According to Wolfram's classification, the following classes of rules are distinguished in order of increasing complexity [5-8]:

First class. The evolution of almost all initial conditions results in fast state stabilization. Any random constructs when these rules are applied disappear.

Second class. As a result of the development of the model, a rapid stabilization of the state occurs. However, in some cases stable fluctuations may occur. Local changes in the initial conditions have a local character for the further course of the evolution of the model.

Third class. For almost all initial conditions, pseudo-random, chaotic sequences arise, and any stable structures are destroyed by the noise surrounding them. It is important that local changes in the initial conditions have a broad, undetectable effect on the course of the entire evolution of the system.

Fourth class. The result of evolution under all the rules are structures that interact in a complex way with the formation of local ones.

Note that definitions of this kind are mostly of a qualitative nature.

Consider the application of the rules for elementary cellular automata.

Take the rule number for example 40 . In binary code it would be $40_{10}=00101000_{2}$.

Let's write the digits of the binary representation of the number into the table:

Table 2. Wolfram's Rule 40

\begin{tabular}{|l|c|c|c|c|c|c|c|c|}
\hline Current state & $\mathbf{1 1 1}$ & $\mathbf{1 1 0}$ & $\mathbf{1 0 1}$ & $\mathbf{1 0 0}$ & $\mathbf{0 1 1}$ & $\mathbf{0 1 0}$ & $\mathbf{0 0 1}$ & $\mathbf{0 0 0}$ \\
\hline The new state of the central cell & 0 & 0 & 1 & 0 & 1 & 0 & 0 & 0 \\
\hline
\end{tabular}

Depending on the states of the neighbor on the left, the cell itself, and the neighbor on the right (the first row of the table), at the next step, the cell will take one of the states indicated in the second row. Let's say for state $101_{2}$ the automaton will go to state 1 . Let's encode the states of the emotional cellular automaton as follows:

1 - "rest";

2 - "aggression".

We will assume that it is possible to influence the emotional state of an individual (cellular automaton) and two of his neighbors ("friends") using a stream of text messages. The current state of the considered emotion machines can be determined, for example, based on the analysis of the closest in time content. We will simulate the state of the individual step by step, or, in another way, step by step, based on the selected rule.

This rule at number 40 according to the Wolfram's classification belongs to the first class of cellular automata. It is characteristic of this class that all cells quickly assume the same state, which becomes stable.

Below in Tables 3-5 are some more examples of rules for elementary emotional cellular automata. 
Applying rule 2 of class number 3 (according to the classification of Wolfram Stephen Wolfram, Stephen) we can quickly stabilize the state of all cells (individuals) and in one of the possible states of «rest» or «aggression», or applying rule 33, we can achieve periodic fluctuations emotions.

Table 3. Wolfram's Rule 30

\begin{tabular}{|l|l|l|l|l|l|l|l|l|}
\hline Current state & $\mathbf{1 1 1}$ & $\mathbf{1 1 0}$ & $\mathbf{1 0 1}$ & $\mathbf{1 0 0}$ & $\mathbf{0 1 1}$ & $\mathbf{0 1 0}$ & $\mathbf{0 0 1}$ & $\mathbf{0 0 0}$ \\
\hline $\begin{array}{l}\text { The new state of the central } \\
\text { cell }\end{array}$ & 0 & 0 & 0 & 1 & 1 & 1 & 1 & 0 \\
\hline
\end{tabular}

Rule 30 exhibits class 3 behavior, which means that the evolution of simple initial conditions leads to chaotic, seemingly random dynamics.

Table 4. Wolfram's Rule 110

\begin{tabular}{|l|c|c|c|c|c|c|c|c|}
\hline Current state & $\mathbf{1 1 1}$ & $\mathbf{1 1 0}$ & $\mathbf{1 0 1}$ & $\mathbf{1 0 0}$ & $\mathbf{0 1 1}$ & $\mathbf{0 1 0}$ & $\mathbf{0 0 1}$ & $\mathbf{0 0 0}$ \\
\hline $\begin{array}{l}\text { The new state of the central } \\
\text { cell }\end{array}$ & 0 & 1 & 1 & 0 & 1 & 1 & 1 & 0 \\
\hline
\end{tabular}

Rule 110 like game Life exhibits Class 4 behavior that is not entirely random, but there is no periodicity. Some of the structures generated by the rule are diverse enough to have Turing completeness $[8,9]$. As a consequence, class 4 systems are in a sense universal.

Table 5. Wolfram's Rule 161

\begin{tabular}{|l|c|c|c|c|c|c|c|c|}
\hline Current state & $\mathbf{1 1 1}$ & $\mathbf{1 1 0}$ & $\mathbf{1 0 1}$ & $\mathbf{1 0 0}$ & $\mathbf{0 1 1}$ & $\mathbf{0 1 0}$ & $\mathbf{0 0 1}$ & $\mathbf{0 0 0}$ \\
\hline $\begin{array}{l}\text { The new state of the central } \\
\text { cell }\end{array}$ & 1 & 0 & 1 & 0 & 0 & 0 & 0 & 1 \\
\hline
\end{tabular}

Rule 161 generates fractal structures, such as nested triangles like this.

An individual can be modeled by more than one emotional cellular automate.

For each set of automata, an own rule and class of rules can be used. In this case, the various states of the collective (stability, fluctuations, cognitive dissonance of the individual).

This approach to modeling the emotional behavior of collectives uses superrouting - signal focusing. Organization of environments and means of rapid dissemination of messages in the form of social networks, subscriptions, audiences of portals, gaming communities, etc. This solves the problem with a great variety: the network of active translators itself adapts the form of the message, filters and distributes to nodes. Signal routing to the entire mass of specific individuals becomes possible, with all the problems.

\section{Discussion}

Taking into account the approach considered in the work, it seems to us that it becomes possible to propose a management model based on viral, multi-agent technologies. In this case, we will specify the method of information delivery. Moreover, in this paradigm, both decentralized and classical hierarchical models can be implemented. If we also consider «sleeping» agents, then it is possible to implement a temporal control model, taking into account the nonlinearity of time in artificial systems [9-14].

\section{Conclusion}


At present, the modeling and dissemination of emotions in human-machine systems can be considered the key to solving many problems of artificial intelligence (various kinds of competitions, modeling creativity, managing teams, etc.).

Especially from the point of view, the application of the considered approach is relevant for solving the problem of modeling the emotional behavior of collectives of individuals. Therefore, the proposed approach to the construction of "emotional" elementary cellular automata and modeling on their basis the behavior of groups of people, the authors seem to be acceptable and of practical value. Along with the emotional, such an approach, from our point of view, is also possible when constructing a "sensitive finite automate".

Thus, having built the appropriate model of the collective and choosing the required model of the emotional cellular automate, we can model the behavior of the collective when applying certain rules $[15,18]$, taking into account the set management goals.

\section{References}

1. S. Moriceau, Cellular automata on a G-set. arXiv: 1105.5335 (2011) [math.DS]

2. M. Margenstern, Small Universal Cellular Automata in Hyperbolic Spaces: A Collection of Jewels (2013)

3. M. Sobottka, D. Gonçalves, A note on the definition of siding block codes and the CurtisHedlund-Lyndon Theorem. arXiv: 1507.02180 [math.DS] (2015)

4. S. Wacker, Cellular Automata on Group Sets and the Uniform Curtis-Hedlund-Lyndon Theorem. In Cellular Automata and Discrete Complex Systems. AUTOMATA (2016) DOI: 10.1007/978-3-319-39300-1_15.

5. T. Ceccherini-Silberstein, M. Coornaert, Cellular Automata and Groups (2010) DOI: 10.1007/978-3-642-14034-1. ISBN: 978-3-642-14033-4, 978-3-642-14034-1

6. S. Wolfram, I am an information pack rat. New Scientist (2014)

7. M. Peragine, The Universal Mind: The Evolution of Machine Intelligence and Human Psychology, Xiphias Press (2016)

8. C. A. Pickover, C. A. Pickover, The Math Book: From Pythagoras to the 57th Dimension, 250 Milestones in the History of Mathematics. Sterling Publishing Company, Inc, (2009) ISBN 978-1402757969.

9. A. G. Hoekstra, J. Kroc, P. Sloot, Simulating complex systems by cellular automata. Springer (2010) ISBN 978-3-642-12202-6

10. L. Manukyan, S. A. Montandon, A. Fofonjka, S. Smirnov, M. C. Milinkovitch. A living mesoscopic cellular automaton made of skin scales. Nature, 544, 173-179 (2017) doi:10.1038/nature22031.

11. W. Peak, M. Messinger, Evidence for complex, collective dynamics and emergent, distributed computation in plants. Proceedings of the National Institute of Science of the USA: journal, 101(4), 918-922 (2004) doi:10.1073/pnas.0307811100. — Bibcode: 2004PNAS..101..918P. - PMID 14732685.

12. A. Deutsch, S. Dormann, Biological Applications. Cellular Automaton Modeling of Biological Pattern Formation. Springer Science. Business Media (2017) ISBN 978-1-48997980-3

13. K. G. F. Janssens, An introductory review of cellular automata modeling of moving grain boundaries in polycrystalline materials. Mathematics and Computers in Simulation, 80, 1361-1381 (2010) doi:10.1016/j.matcom.2009.02.011 
14. H. Gerard't, The Cellular Automaton Interpretation of Quantum Mechanics. Springer International Publishing Springer (2016) ISBN 978-3-319-41285-6, 978-3-319-41284-9.

15. V. E. Meshkov, E. V. Meshkova, V. S. Churakov, Finite emotional automata, Natural and technical sciences, 12(114), 299-305 (2017)

16. Chaos and Cyber Culture Timothy Leary. Ronin Publishing. ISBN: 0914171771 https://www.livelib.ru/book/1002696959-chaos-and-cyber-culture-timothy-leary (Last accessed 11.06.2020)

17. S. Wolfram, "Computation theory of cellular automata". Communications in Mathematical Physics, 96(1), 15-57 (1984) Bibcode:1984CMaPh..96...15W. doi:10.1007/BF01217347. S2CID 121021967

18. V. Meshkov, N. Kochkovaya, I. Usova, Formation of functional-role communication clusters based on morphological features of the verbal context. XIII International Scientific and Practical Conference "State and Prospects for the Development of Agribusiness - INTERAGROMASH 2020” E3S Web Conf. 175 (2020) DOI https://doi.org/10.1051/e3sconf/202017515007 